\title{
Desarrollo de una herramienta numérica de análisis para losas de hormigón armado sometidas a aceleraciones verticales sísmicas
}

\section{Development of a numerical tool for the analysis of reinforced concrete slabs under seismic vertical accelerations}

\section{Deni Herrera}

Fecha de entrega: 25 de agosto 2011 Fecha de aceptación: 22 de noviembre 2011

Constructora El Sauce, Av. General Borgoño 934, oficina 301, Antofagasta, Chile, dherrera@elsauce.cl

Durante el terremoto en el centro sur de Chile del 27 de febrero del 2010 muchas estructuras fueron solicitadas fuertemente debido a aceleraciones horizontales considerables, sin embargo, la componente vertical de aceleración también fue importante. Esta característica ha motivado este trabajo, donde se estudia el efecto que produce la componente vertical de aceleración sobre losas de hormigón armado, teniendo en cuenta que la aceleración se aplica sobre el borde de la losa. La metodología utilizada contempló la elaboración de un programa computacional escrito en lenguaje MATLAB. Este programa utiliza el MEF y permite calcular tanto las tensiones como deformaciones en la losa. Inicialmente se realizó un análisis estático consistente en obtener las distribuciones de tensiones que genera una carga estática uniformemente distribuida de acuerdo a la norma NCh 1537.Of86. Luego, se realizó un análisis sísmico consistente en obtener las envolventes de tensiones que genera la aplicación del registro vertical de aceleraciones de San Pedro de la Paz del terremoto del 27/02/2010. Se presenta el contexto teórico, la formulación del MEF y la validación numérica de los resultados. Posteriormente se analizan 2 losas de distinta geometría con el propósito de obtener las distribuciones de tensiones para el caso estático y envolventes de tensiones para el caso sísmico; al igual que las frecuencias y periodos naturales de cada losa. Los resultados revelan que las tensiones inducidas por las cargas estáticas son mayores a las inducidas por las cargas sísmicas para la losa con un periodo fundamental de $0.031 \mathrm{~s}$. Mientras que para la losa con un periodo fundamental de 0.104 s resultó lo contrario.

Palabras clave: aceleraciones verticales, elementos finitos $M Z C$ y DKT, losas de hormigón armado, placas, discretización
During the Chile Centre-South $27^{\text {th }}$ February 2010 earthquake, many structures were strongly shaken due to considerable horizontal accelerations; however, the vertical acceleration component was important too. This feature has motivated this work, where the vertical acceleration component effect on reinforced concrete slabs is studied, when this acceleration acts on the slab edges. The methodology contemplated a computer software written in MATLAB code. This program uses the FEM and allows the calculation of stresses and strains in the slab. Initially a static analysis was carried out, which consisted of calculating the stress distributions generated by a uniformly distributed static load according to the norm NCh 1537Of.86. Subsequently, the seismic analysis consisted of obtaining the stress envelopes caused by the vertical acceleration recording of San Pedro de la Paz for the 27/02/2010 earthquake. The theoretical framework, the FEM formulation and the numerical validation of the results are presented. Then, two slabs with different geometry are analysed with the purpose of estimating the stress distributions for the static case and the stress envelopes for the seismic case; as well as the natural frequencies and periods for each slab. The results reveal that stresses induced by the static loads are higher than the induced by the seismic loading for the slab with a fundamental period of $0.031 \mathrm{~s}$, whilst the contrary resulted for the slab with a fundamental period of $0.104 \mathrm{~s}$.

Keywords: vertical accelerations, MZC and DKT finite elements, reinforced concrete slabs, plates, discretization

varios de sus elementos estructurales. Deformaciones irreversibles se evidenciaron por medio de la aparición de fisuras, grietas e incluso desprendimiento del hormigón y falla del acero de refuerzo. Esto condujo a la falla de importantes elementos estructurales e incluso el colapso de 
algunas estructuras (Betanzo 2010). En general las fallas más importantes suceden en muros, vigas y columnas de hormigón armado, en cambio en las losas los daños son menores. Tal vez debido a esto las losas de hormigón armado son menos estudiadas. Sin embargo, las fotos de la Figura 1 muestran ejemplos de daños en losas ocurridas en el terremoto del 27/02/2010. Por lo tanto, también se hace necesario estudiar el comportamiento sísmico de las losas y en particular el efecto de la componente vertical del movimiento, el cual es aún menos estudiado.
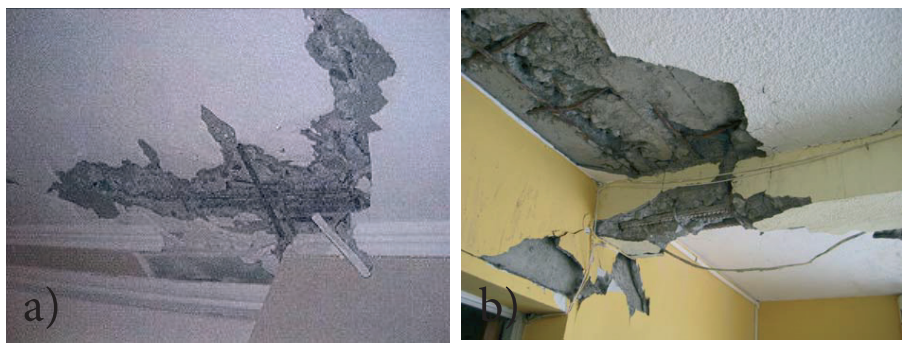

Figura 1: Daños en losa a nivel de esquina de muro a) edificio Don Manuel 21 niveles, Macúl (IDIEM 2010) y b) último piso del edificio Palet de 9 niveles, Concepción

El terremoto del 27/02/2010 se caracterizó por presentar altas aceleraciones verticales, alcanzando en algunos registros valores muy similares a las aceleraciones horizontales (Barrientos 2010; Boroschek et al. 2010). Sin embargo, la componente vertical de la aceleración es raramente considerada en el diseño sismorresistente de edificios y menos aún en el diseño sismorresistente de losas de hormigón armado.

Estos valores significativos de aceleración vertical merecen un estudio especial respecto a la respuesta que puedan originar en las superestructuras. Uno de los objetivos de este trabajo apunta a poder contribuir a las recomendaciones dadas por los códigos de diseño sismorresistentes respecto a la componente vertical del terremoto de diseño. Por ejemplo, la norma Chilena NCh 4330f96, la cual rige el diseño sismorresistente de edificios, no considera la componente vertical del terremoto de diseño, ya que solo se indica que las solicitaciones sísmicas relevantes provienen de aceleraciones horizontales.

\section{Ecuación diferencial de placas}

La expresión que permite estimar la deformación vertical de una placa y que puede ser usada para calcular la flecha de una losa de hormigón a partir de las propiedades geométricas y resistentes de este material y las cargas aplicadas viene dada por (Timoshenko, 1937; Chakraverty 2009):

$$
\bar{D}_{f}\left(\frac{\partial^{4} w}{\partial x^{4}}+2 \frac{\partial^{4} w}{\partial x^{2} \partial y^{2}}+\frac{\partial^{4} w}{\partial y^{4}}\right)+\bar{m} \frac{\partial^{2} w}{\partial t^{2}}=-q
$$

La expresión (1) es una ecuación diferencial de cuarto orden que relaciona la flecha $w$ con la carga repartida $q \mathrm{y}$ las propiedades del material. Donde $w$ es el desplazamiento vertical o flecha, $\bar{m}$ es la masa por unidad de superficie y $q$ es la carga distribuida sobre la losa. El parámetro $\bar{D}_{f}$ representa la rigidez flexural de la placa y su expresión está dada por:

$$
\bar{D}_{f}=\frac{E t^{3}}{12\left(1-v^{2}\right)}
$$

donde $E$ es el módulo de Young o de elasticidad del material, $v$ es la relación de Poisson y $t$ es el espesor de la losa.

\section{Discretización de la losa por medio del MEF}

Para resolver (1) se ha utilizado el Método de los Elementos Finitos MEF. Para ello se han escogido dos elementos en particular: el elemento rectangular MZC (Melosh 1963, Zienkievicz y Cheung 1967) y el elemento triangular DKT (Discrete Kirchhoff Triangular, ver Batoz et al. 1980). La diferencia que existe entre estos dos elementos, es que el elemento triangular proporciona más versatilidad geométrica que el elemento rectangular, por ejemplo se pueden realizar mallados con geometrías de losas circulares.

Al discretizar la losa y al aplicar el MEF sobre (1), se obtienen matrices de rigidez y masa, tanto para el elemento MZC como para el elemento DKT. Una vez que han sido calculadas las matrices de rigidez y masa tanto para el elemento MZC como para el elemento DKT, la ecuación que permite encontrar los desplazamientos de la losa es,

$$
[\mathrm{M}]\{\ddot{\mathrm{u}}\}+[\mathrm{C}]\{\dot{\mathrm{u}}\}+[\mathrm{K}]\{\mathrm{u}\}=-[\mathrm{M}]\{1\} \ddot{\mathrm{y}}_{\mathrm{s}}(\mathrm{t})
$$

La expresión (3) es una ecuación de movimiento para sistemas de múltiples grados de libertad. Esta ecuación matricial de movimiento posee la característica de involucrar las matrices de masa $[\mathrm{M}]$, amortiguación $[\mathrm{C}]$ y rigidez $[\mathrm{K}]$ de la losa. Cabe destacar que los coeficientes 
que componen la matriz de amortiguación no pueden ser obtenidos explícitamente, debido a esto se incorporan en las ecuaciones de movimiento solo a nivel modal (Paz 1992). También se debe mencionar que (3) es un sistema de ecuaciones acoplado, por lo que para resolverlo se hace necesario desacoplarlo a través del Método de Superposición Modal (Paz 1992). Al aplicar el Método de Superposición Modal sobre (3) se obtiene la siguiente ecuación modal de movimiento,

$$
\ddot{\mathrm{g}}_{\mathrm{i}}+2 \xi_{\mathrm{i}} \omega_{\mathrm{i}} \dot{\mathrm{g}}_{\mathrm{i}}+\omega_{\mathrm{i}}^{2} \mathrm{~g}_{\mathrm{i}}=\ddot{\mathrm{y}}_{\mathrm{s}}(\mathrm{t}) \quad ; \quad \mathrm{i}=1,2,3, \ldots \ldots, \mathrm{n}
$$

La expresión (4) ha sido resuelta por el método de Newmark (1959), donde el lado derecho corresponde al registro vertical de aceleraciones del terremoto del 27/02/2010 obtenido en el Colegio Concepción de San Pedro de la Paz (Barrientos 2010).

\section{Matriz de rigidez y masa del elemento MZC}

La Figura 2 muestra el elemento rectangular de cuatro nodos denominado MZC, el cual se basa en la siguiente aproximación,

$$
\begin{aligned}
& w=\alpha_{1}+\alpha_{2} x+\alpha_{3} y+\alpha_{4} x^{2}+\alpha_{5} x y+\alpha_{6} y^{2}+\alpha_{7} x^{3} \\
& +\alpha_{8} x^{2} y+\alpha_{9} x y^{2}+\alpha_{10} y^{3}+\alpha_{11} x^{3} y+\alpha_{12} x y^{3}
\end{aligned}
$$

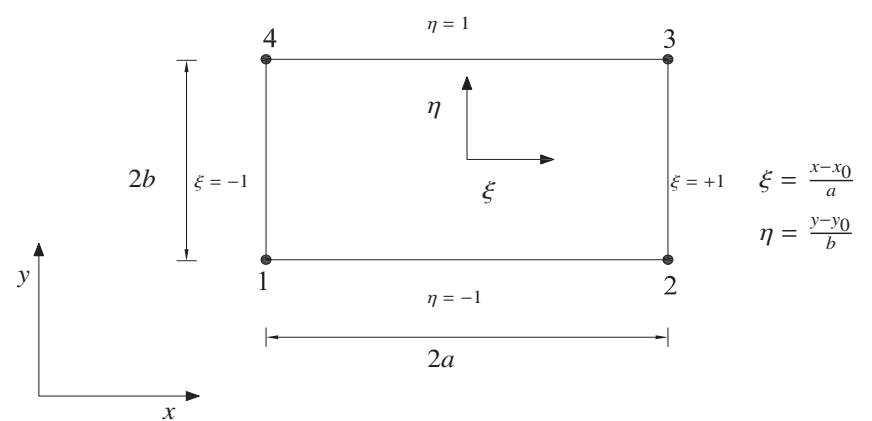

Figura 2: Elemento de placa rectangular de cuatro nodos no conforme

La aproximación (5) ha sido resuelta por Melosh (1963) y Zienkiewicz y Cheung (1967), entregando la siguiente expresión para la flecha $w$,

$$
w=\sum_{i=1}^{4}\left[N_{i} w_{i}+\bar{N}_{i}\left(\frac{\partial w}{\partial x}\right)_{i}+\overline{\bar{N}}_{i}\left(\frac{\partial w}{\partial y}\right)_{i}\right]=N a^{(e)}
$$

$$
\begin{aligned}
& N=\left[N_{1} N_{2} N_{3} N_{4}\right] \quad ; \quad N_{i}=\left\lfloor N_{i} \bar{N}_{i} \overline{\bar{N}}_{i}\right\rfloor \\
& a^{(e)}=\left[\begin{array}{l}
a_{1}^{(e)} \\
a_{2}^{(e)} \\
a_{3}^{(e)} \\
a_{4}^{(e)}
\end{array}\right] ; \quad a_{i}^{(e)}=\left[w_{i}\left(\frac{\partial w}{\partial x}\right)_{i}\left(\frac{\partial w}{\partial y}\right)_{i}\right]^{T}
\end{aligned}
$$

donde $N$ son las matrices de funciones de forma y $a$ es el vector de movimientos del elemento $e$ de un nodo $i$, respectivamente. Las expresiones analíticas de las funciones de forma $N_{i}=\left[N_{i}, \overline{N_{i}}, \overline{\overline{N_{i}}}\right]$ en coordenadas naturales son,

$$
\begin{aligned}
& N_{i}=\frac{1}{8}\left(1+\xi_{i} \xi\right)\left(1+\eta_{i} \eta\right)\left(2+\xi_{i} \xi+\eta_{i} \eta-\xi^{2}-\eta^{2}\right) \\
& \bar{N}_{i}=\frac{a}{8}\left(\xi^{2}-1\right)\left(\xi+\xi_{i}\right)\left(1+\eta_{i} \eta\right) \\
& \overline{\bar{N}}_{i}=\frac{b}{8}\left(\eta^{2}-1\right)\left(\eta+\eta_{i}\right)\left(1+\xi_{i} \xi\right)
\end{aligned}
$$

La matriz de deformación generalizada de flexión se obtiene a partir del vector de deformaciones generalizadas de flexión de la siguiente manera,

con

$$
\hat{\varepsilon}_{f}=\left[\begin{array}{c}
-\frac{\partial^{2} w}{\partial x^{2}} \\
-\frac{\partial^{2} w}{\partial y^{2}} \\
-\frac{\partial^{2} w}{\partial x \partial y}
\end{array}\right]=\sum_{i=1}^{4} B_{i} a_{i}^{(e)}=B a^{(e)}
$$

y

$$
B_{i}=\left[\begin{array}{rrr}
-\frac{\partial N_{i}}{\partial x^{2}} & -\frac{\partial \bar{N}_{i}}{\partial x^{2}} & -\frac{\partial^{2} \overline{\bar{N}}_{i}}{\partial x^{2}} \\
-\frac{\partial^{2} N_{i}}{\partial y^{2}} & -\frac{\partial \bar{N}_{i}}{\partial y^{2}} & -\frac{\partial^{2} \bar{N}_{i}}{\partial y^{2}} \\
-2 \frac{\partial^{2} N_{i}}{\partial x \partial y} & -2 \frac{\partial \bar{N}_{i}}{\partial x \partial y} & -2 \frac{\partial^{2} \overline{\bar{N}}_{i}}{\partial x \partial y}
\end{array}\right]
$$

La matriz de rigidez elemental viene dada por:

$$
K_{M Z C}^{(e)}=\iint_{A^{(e)}} B_{i}^{T} \hat{D}_{f} B_{i} d x d y
$$

donde

$$
\hat{D}_{f}=\frac{E}{1-v^{2}}\left[\begin{array}{ccc}
1 & v & 0 \\
v & 1 & 0 \\
0 & 0 & \frac{1-v}{2}
\end{array}\right]
$$


es la matriz constitutiva de flexión para un material elástico isótropo. La matriz de masa elemental es,

$$
M_{M Z C}^{(e)}=\bar{m} \iint_{A^{(e)}} N_{i}^{T} N_{i} d x d y
$$

La expresión del vector de fuerzas nodales equivalentes para una carga $q$ uniformemente distribuida sobre el elemento $e$ es:

$$
q_{i}^{(e)}=4 q a b\left[\begin{array}{llllllllllll}
\frac{1}{4} & \frac{a}{12} & \frac{b}{12} & \frac{1}{4} & -\frac{a}{12} & \frac{b}{12} & \frac{1}{4} & -\frac{a}{12} & -\frac{b}{12} & \frac{1}{4} & \frac{a}{12} & -\frac{b}{12}
\end{array}\right] .
$$

Las integrales (13) y (15) se calculan por el método de integración numérica de Gauss para dos dimensiones (Oñate 1992), obteniendo así la matriz de rigidez y de masa elemental. Las matrices en coordenadas globales de rigidez y de masa, al igual que el vector en coordenadas globales de fuerzas nodales, se obtienen por medio de un proceso de ensamblaje a través de los vectores de coordenadas y conectividades de cada elemento que compone la malla de elementos rectangulares. Los esfuerzos en los puntos nodales se calculan con la siguiente expresión,

$$
\left[\begin{array}{l}
\sigma_{x} \\
\sigma_{y} \\
\tau_{x y}
\end{array}\right]^{(e)}=D_{f} B a^{(e)}
$$

Debido a que las tensiones se calculan en los nodos de cada elemento, puede ocurrir que en nodos comunes sus valores varíen considerablemente. Esto se puede solucionar promediando los valores de las tensiones que comparten el mismo nodo, lo cual se denomina alisado de tensiones. Una vez que se ha resuelto (1) y se han utilizado (3) y (4) se pueden obtener las tensiones en la losa a través de (17). Cabe destacar que se utiliza (17) para el cálculo de tensiones del caso estático y sísmico del elemento rectangular.

\section{Matriz de rigidez y masa del elemento DKT}

La formulación del elemento DKT se basa en la teoría discreta de Kirchhoff para placas delgadas, la que da origen al elemento triangular discreto de Kirchhoff (Figura 3). El elemento DKT ha demostrado tener un excelente comportamiento con respecto a su convergencia (Batoz et al. 1980).

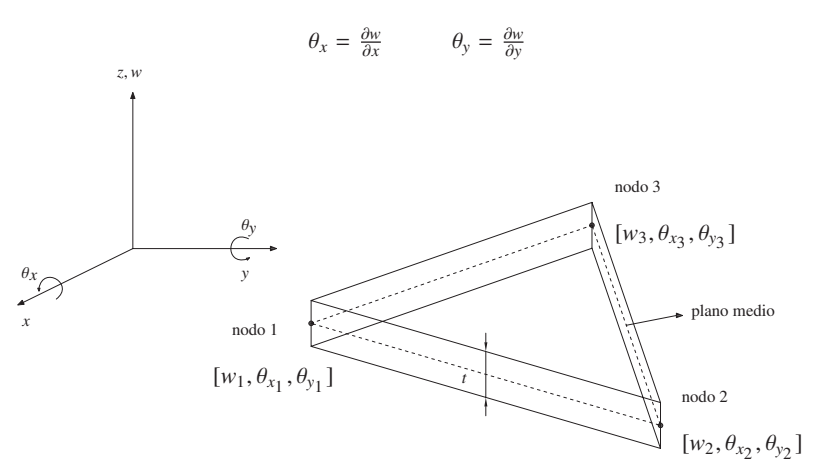

Figura 3: Definición geométrica del elemento de placa DKT y convenio de signos para desplazamientos y giros

Para placas delgadas la deformación transversal por corte y, por lo tanto su energía de deformación $U_{S}$, es despreciable comparado con la energía de deformación por flexión $U$. Teniendo en cuenta lo anterior, la formulación de la matriz de rigidez del elemento DKT se basa en la siguiente expresión,

$$
U=\frac{1}{2} \int_{A} \varepsilon_{f}^{T} \hat{D}_{f} \varepsilon_{f} d x d y
$$

donde $U$ es la energía de deformación por flexión, $A$ es el área del plano medio del elemento, $\varepsilon_{f}$ es el vector de deformaciones por flexión y $\hat{D_{f}}$ es la matriz constitutiva. No se considera la deformación por corte ya que para placas delgadas el aporte energético de la deformación por corte transversal es despreciable. Además (18) contiene solamente las primeras derivadas de $\theta_{x}$ y $\theta_{y}$, por lo tanto es posible establecer funciones de interpolación que satisfagan los requerimientos de compatibilidad, relacionando la rotación de la normal $\left(\theta_{x}, \theta_{y}\right)$ a la superficie media con los desplazamientos transversales $w$. Este objetivo se alcanza con las siguientes consideraciones:

a. El elemento triangular debe tener solo 9 grados de libertad, los cuales son el desplazamiento $w$ y los giros $\theta_{x}$ y $\theta_{y}$ de los tres nodos esquineros.

b. Los giros nodales deben ser $\theta_{x}=+\frac{\partial w}{\partial x}$ y $\theta_{y}=+\frac{\partial w}{\partial y}$, de modo que se satisfagan las condiciones de borde del elemento.

c. Ya que los modelos de elementos de placas delgadas son gobernados por la teoría de Kirchhoff, ésta se puede aplicar sobre cualquier punto discreto del elemento.

d. La compatibilidad de los giros $\theta_{x}$ y $\theta_{y}$ no debe perderse. 
El punto de partida para obtener la matriz de rigidez del elemento de 3 nodos DKT, es el elemento triangular de placa de Reissner-Mindlin de 6 nodos mostrado en la Figura 4, sometido a varias condiciones y/o consideraciones (Batoz et al. 1980). a) Elemento inicial

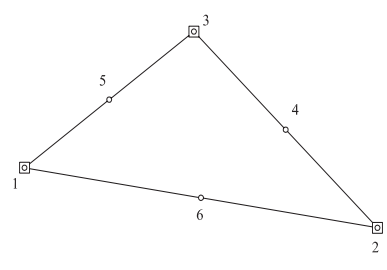

Variables nodales: a) Elemento DKT final

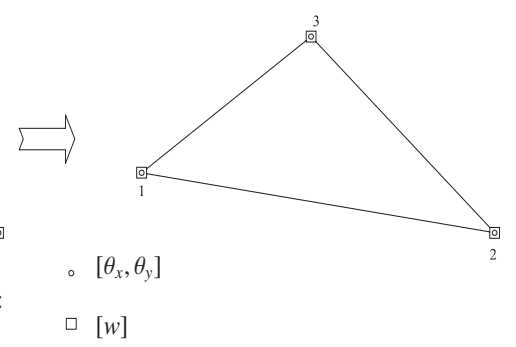

Figura 4: Elemento de placa DKT de tres nodos

Utilizando las consideraciones anteriores se puede establecer una interpolación del desplazamiento $w$ (flecha) y los giros nodales $\theta_{x}$ y $\theta_{y}$, a través de,

$$
\begin{gathered}
u=N a^{(e)} \\
u=\left[\begin{array}{lll}
w & \theta_{x} & \theta_{y}
\end{array}\right]^{T}
\end{gathered}
$$

donde $u$ es el vector de movimientos y $a$ es el vector de movimientos de cada grado de libertad del elemento,

$$
a^{(e)}=\left[\begin{array}{lllllllll}
w_{1} & \theta_{x 1} & \theta_{y 1} & w_{2} & \theta_{x 2} & \theta_{y 2} & w_{3} & \theta_{x 3} & \theta_{y 3}
\end{array} \mid\right.
$$

$N=\left\{\begin{array}{l}N_{w} \\ N_{\theta_{k}} \\ N_{\theta_{y}}\end{array}\right\}=\left[\begin{array}{lllllllll}N_{w 1} & N_{w 2} & N_{w 3} & N_{w 4} & N_{w 5} & N_{w 6} & N_{w 7} & N_{w 8} & N_{w 9} \\ N_{\theta_{x 1}} & N_{\theta_{x 2}} & N_{\theta_{x 3}} & N_{\theta_{x 4}} & N_{\theta_{x 5}} & N_{\theta_{x 6}} & N_{\theta_{x 7}} & N_{\theta_{x 8}} & N_{\theta_{x 9}} \\ N_{\theta_{y 1}} & N_{\theta_{y 2}} & N_{\theta_{y 3}} & N_{\theta_{y 4}} & N_{\theta_{y 5}} & N_{\theta_{y 6}} & N_{\theta_{y 7}} & N_{\theta_{y 8}} & N_{\theta_{y 9}}\end{array}\right]$

$N$ es la matriz de funciones de forma del elemento DKT que aproximan o interpolan los desplazamientos y giros nodales. La matriz de deformación generalizada de flexión se obtiene a partir del vector $\varepsilon_{f}$ y (21) como,

$$
\varepsilon_{f}=B_{f} a^{(e)},
$$

donde $B_{f}$ es la matriz de deformación generalizada del elemento definida por:

$$
B_{f}=\frac{1}{2 A}\left[\begin{array}{c}
y_{31} \frac{\partial N_{\theta_{x}}^{T}}{\partial \xi}+y_{12} \frac{\partial N_{\theta_{y}}^{T}}{\partial \eta} \\
-x_{31} \frac{\partial N_{\theta_{x}}^{T}}{\partial \xi}-x_{12} \frac{\partial N_{\theta_{y}}^{T}}{\partial \eta} \\
-x_{31} \frac{\partial N_{\theta_{x}}^{T}}{\partial \xi}-x_{12} \frac{\partial N_{\theta_{x}}^{T}}{\partial \eta}+y_{31} \frac{\partial N_{\theta_{y}}^{T}}{\partial \xi}+y_{12} \frac{\partial N_{\theta_{y}}^{T}}{\partial \eta}
\end{array}\right]
$$

con $2 \mathrm{~A}=x_{31} y_{12}-x_{12} y_{31}$ y teniendo en cuenta que $A$ es el área del elemento triangular. Las derivadas de $N_{\theta_{x}} \mathrm{y} N_{\theta_{y}}$ con respecto a $\xi$ y $\eta$ se pueden encontrar explícitamente en Batoz et al. (1980), al igual que las expresiones de los coeficientes $x_{i j}$ e $y_{i j}$ La expresión para encontrar la matriz de rigidez del elemento DKT es la siguiente,

$$
K_{D K T}^{(e)}=2 A^{(e)} \int_{0}^{1} \int_{0}^{1-\eta} B_{f}^{T} \hat{D_{f}} B_{f} d \xi d \eta
$$

y para la matriz de masa se tiene de acuerdo a Luo y Hutton (2002) que,

$$
M_{D K T}^{(e)}=2 A^{(e)} \bar{m} \int_{0}^{1} \int_{0}^{1-\eta} N_{w}^{T} N_{w} d \xi d \eta
$$

Finalmente, la expresión del vector de fuerzas nodales equivalente para una carga $q$ uniformemente distribuida sobre el elemento es,

$$
q_{i}^{(e)}=\frac{q A^{(e)}}{3}\left[\begin{array}{lllllllll}
1 & 0 & 0 & 1 & 0 & 0 & 1 & 0 & 0
\end{array}\right]
$$

donde $q$ es la carga repartida sobre el elemento triangular y $A$ es el área del elemento triangular. Al igual que para el elemento rectangular, las ecuaciones (25) y (26) se calculan por el método de integración numérica de Gauss para dos dimensiones (Oñate 1992), obteniendo así la matriz de rigidez y de masa elemental. Las matrices en coordenadas globales de rigidez y de masa, al igual que el vector en coordenadas globales de fuerzas nodales, se obtienen con el proceso de ensamblaje a través de la matriz de transformación de coordenadas, y también, por los vectores de coordenadas y conectividades de cada elemento que compone la malla de elementos triangulares. Los esfuerzos en los puntos nodales se calculan con la siguiente expresión,

$$
\left[\begin{array}{l}
\sigma_{x} \\
\sigma_{y} \\
\tau_{x y}
\end{array}\right]^{(e)}=D_{f} B_{f} a^{(e)}
$$


Nuevamente se tiene que las tensiones calculadas en los nodos de cada elemento puede causar que en nodos comunes sus valores varíen. Esto se puede solucionar usando alisado de tensiones. Como también se dijo anteriormente, una vez que se ha resuelto (1) y se han utilizado (3) y (4) se pueden obtener las tensiones sobre la losa a través de (28). Cabe destacar que (28) se ha utilizado para el cálculo de tensiones en el caso estático y sísmico del elemento triangular.

\section{Validación numérica}

Para poder utilizar el código programado, primero se ha validado, es decir, se comparan los resultados del programa con las soluciones analíticas o exactas conocidas, las cuales se han basado en la teoría de la elasticidad lineal, y específicamente en la teoría de placas y en la teoría de dinámica estructural. Debido a que el programa involucra el comportamiento sísmico de una estructura (respuesta de una losa bajo la acción de la componente vertical de aceleraciones), ha sido necesario obtener las matrices de rigidez y masa para la resolución de la ecuación de movimiento. La matriz de rigidez de la losa se ha validado con el caso de una losa empotrada en todos sus bordes y sometida a una carga repartida $q$. Y la matriz de masa, mediante la comparación de las frecuencias naturales entregadas por una expresión analítica proveniente de la teoría de vibración libre de placas con las que entrega el programa elaborado con el MEF. Finalmente, la integración en el tiempo del registro de aceleraciones, se ha validado comparando la respuesta de un oscilador de un grado de libertad, obtenida mediante su solución analítica con la respuesta que otorga el método numérico de Newmark (1959).

\section{Validación del caso estático}

En este caso se estudia la convergencia de la flecha central de una losa cuadrada con todos sus bordes empotrados. La solución analítica del problema, según la teoría de Kirchhoff para placas delgadas, está dada por la siguiente expresión (Novoa 2005),

$$
w_{\text {centro }}=0.01512 \frac{q L^{4}\left(1-v^{2}\right)}{E t^{3}},
$$

donde $w$ es la flecha central, $q$ es la carga repartida, $L$ es el largo, $E$ es el módulo de elasticidad estático, $v$ es la razón de Poisson y $t$ es el espesor de la losa. Las propiedades de la losa con las que se ha realizado el análisis y la comparación de los resultados se muestran la Tabla 1 y en la Figura 5.

Tabla 1: Propiedades de la losa usadas en la validación

\begin{tabular}{|c|c|}
\hline$M[\mathrm{~kg}]$ & 100 \\
\hline$C\left[\frac{N s}{m}\right]$ & 50 \\
\hline$K\left[\frac{N}{m}\right]$ & 250000 \\
\hline$\xi$ & 0.05 \\
\hline$F(t)$ & $98 \operatorname{sen}\left(\omega_{0} t\right)$ \\
\hline$\omega_{0}\left[\frac{\text { Rad }}{s}\right]$ & 10 \\
\hline
\end{tabular}

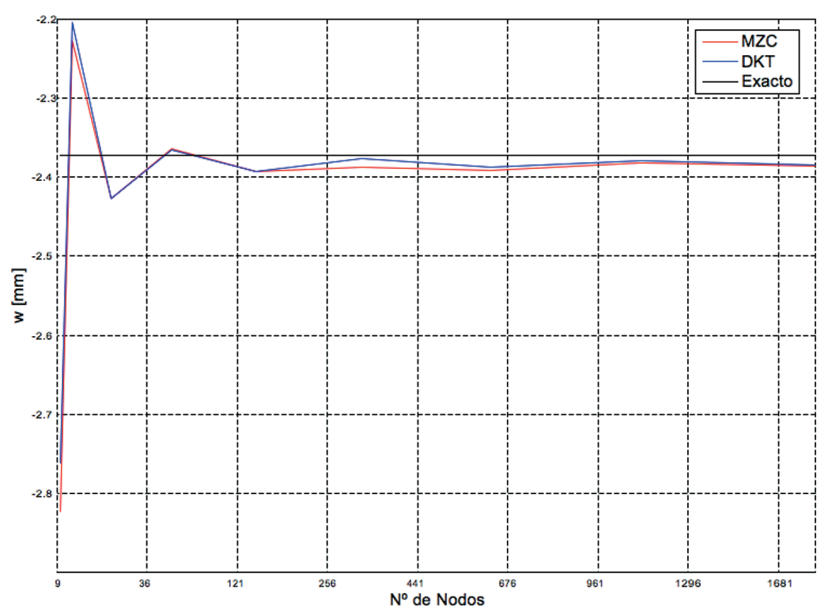

Figura 5: Convergencia del elemento MZC y DKT para losas rectangulares en el caso estático.

De la Figura 5 se puede deducir que tanto el elemento MZC como DKT entregan aproximaciones razonables de la solución analítica de la flecha central de la losa. En las Figuras 6 y 7 se muestran los resultados de las deformadas de la losa con el elemento MZC v DKT.

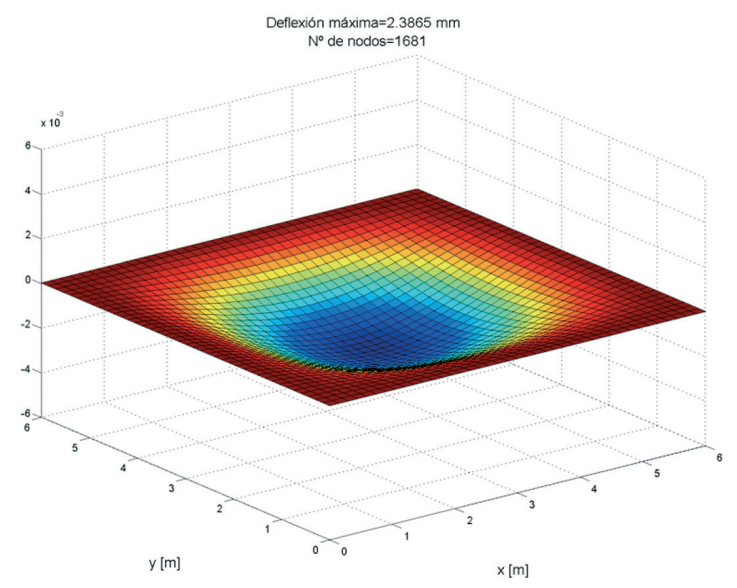

Figura 6: Deformada de la losa discretizada con elementos rectangulares 


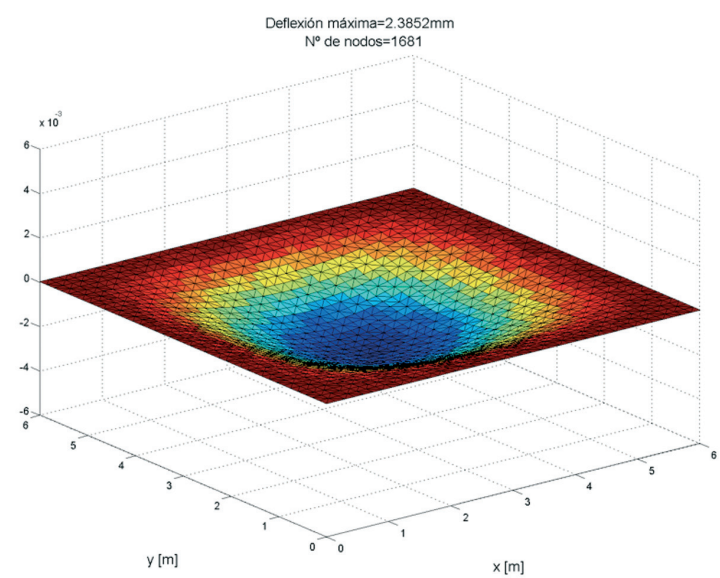

Figura 7: Deformada de la losa discretizada en 1681 nodos con elementos triangulares

\section{Validación de caso dinámico}

La validación del caso dinámico se ha llevado a cabo a través de la comparación de las 6 primeras frecuencias naturales obtenidas de la teoría de vibración libre de placas y las obtenidas a través del programa. Cabe destacar que la losa que se ha utilizado para la validación es una losa cuadrada simplemente apoyada. Existe una expresión teórica para las frecuencias naturales válida para estos tipos de losas dada por (Timoshenko, 1937; Chakraverty 2009):

$$
\omega_{m}=\pi^{2} \sqrt{\bar{D}_{f} \rho t}\left[\left(\frac{m}{a}\right)^{2}+\left(\frac{m}{b}\right)^{2}\right]
$$

donde $\bar{D}_{f}$ es la rigidez flexural de una placa, $\rho$ es la densidad del material de la placa, $t$ es el espesor de la placa, $a$ y $b$ son las longitudes de los lados de la placa, y finalmente, $m$ indica la numeración de los modos de vibrar de la placa. La Tabla 1 presenta los valores de las propiedades de la losa usados en los cálculos numéricos.

Tabla 2: Valores utilizados en el programa en el caso dinámico

\begin{tabular}{|c|c|}
\hline Condiciones de borde & simplemente apoyada en todos sus extremos \\
\hline Espesor $t[\mathrm{~m}]$ & 0,15 \\
\hline$E\left[\frac{N}{\mathrm{~m}^{2}}\right]$ & $2,302581 \times 10^{10}$ \\
\hline$v$ & 0,2 \\
\hline$\rho\left[\frac{\mathrm{kg}}{3}\right]$ & 2500 \\
\hline $\bar{D}_{f}\left[\frac{N}{\mathrm{~m}^{2}}\right]$ & 6745842.7 \\
\hline
\end{tabular}

Las Tablas 2, 3 y 4 presentan los resultados obtenidos de las frecuencias naturales, en donde la letra $m$ indica el número de frecuencia natural calculada.
Tabla 3: Frecuencias naturales en rad/s para una losa de 1 $\mathrm{m}$ x $1 \mathrm{~m}$ para distintas discretizaciones

\begin{tabular}{|c|c|c|c|c|c|c|c|c|c|c|c|}
\hline \multirow{3}{*}{$\mathrm{m}$} & \multicolumn{10}{|c|}{$\mathrm{N}^{0}$ de nodos } & \multirow{3}{*}{ Exacto } \\
\hline & \multicolumn{2}{|c|}{121} & \multicolumn{2}{|c|}{256} & \multicolumn{2}{|c|}{441} & \multicolumn{2}{|c|}{676} & \multicolumn{2}{|c|}{961} & \\
\hline & MZC & DKT & MZC & DKT & MZC & DKT & MZC & DKT & MZC & DKT & \\
\hline 1 & 2634.7 & 2690.4 & 2641.7 & 2666.5 & 2644,2 & 2658.2 & 2645.4 & 2654.3 & 2646.0 & 2652.2 & 2647.4 \\
\hline 2 & 10396 & 11254 & 10500 & 10882 & 10539 & 10758 & 10557 & 10698 & 10567 & 10666 & 10589.9 \\
\hline 3 & 22927 & 26811 & 23391 & 25107 & 23574 & 24609 & 23663 & 24348 & 23712 & 24196 & 23827.2 \\
\hline 4 & 39833 & 49772 & 41055 & 45429 & 41584 & 44415 & 41850 & 43808 & 42000 & 43425 & 42359.6 \\
\hline 5 & 60855 & 78741 & 63219 & 71583 & 64368 & 70072 & 64975 & 69065 & 65326 & 68395 & 66186.9 \\
\hline 6 & 85962 & 109440 & 86159 & 104780 & 85995 & 99790 & 85977 & 94992 & 85983 & 92282 & 95309.1 \\
\hline
\end{tabular}

Tabla 4: Frecuencias naturales en rad/s para una losa de 6 $\mathrm{m} \times 6 \mathrm{~m}$ para distintas discretizaciones

\begin{tabular}{|c|c|c|c|c|c|c|c|c|c|c|c|}
\hline \multirow{3}{*}{$\mathrm{m}$} & \multicolumn{10}{|c|}{$\mathrm{N}^{\circ}$ de nodos } & \multirow{3}{*}{ Exacto } \\
\hline & \multicolumn{2}{|c|}{121} & \multicolumn{2}{|c|}{256} & \multicolumn{2}{|c|}{441} & \multicolumn{2}{|c|}{676} & \multicolumn{2}{|c|}{961} & \\
\hline & MZC & DKT & MZC & DKT & MZC & DKT & MZC & DKT & MZC & DKT & \\
\hline 1 & 73,1 & 74.7 & 73.3 & 74.0 & 73.4 & 73.8 & 73.4 & 73.7 & 73,50 & 73.67 & 73.5 \\
\hline 2 & 288.7 & 312,6 & 291.6 & 302.2 & 292.7 & 298.8 & 293.2 & 297.1 & 293.52 & 296.26 & 294.1 \\
\hline 3 & 636.8 & 744.7 & 649.7 & 697.4 & 654.8 & 683.5 & 657.2 & 676.3 & 658.66 & 672.12 & 661.8 \\
\hline 4 & 1106.5 & 1382.6 & 1140.4 & 1261.9 & 1155.1 & 1233.7 & 1162.5 & 1216.9 & 1166.67 & 1206.24 & 1176.6 \\
\hline 5 & 1690.4 & 2187.2 & 1756.1 & 1988.4 & 1788 & 1946.4 & 1804.8 & 1918.5 & 1814.61 & 1899.85 & 1838.5 \\
\hline 6 & 2387.8 & 3040.1 & 2393.3 & 2910.6 & 2388.8 & 2772 & 2388.2 & 2638.7 & 2388.42 & 2563.38 & 2647.4 \\
\hline
\end{tabular}

Tabla 5: Frecuencias naturales en rad/s para una losa de $12 \mathrm{~m} \mathrm{x}$ $12 \mathrm{~m}$ para distintas discretizaciones

\begin{tabular}{|c|c|c|c|c|c|c|c|c|c|c|c|}
\hline \multirow{3}{*}{$\mathrm{m}$} & \multicolumn{10}{|c|}{$\mathbf{N}^{0}$ de nodos } & \multirow{3}{*}{ Exacto } \\
\hline & \multicolumn{2}{|c|}{121} & \multicolumn{2}{|c|}{256} & \multicolumn{2}{|c|}{441} & \multicolumn{2}{|c|}{676} & \multicolumn{2}{|c|}{961} & \\
\hline & MZC & DKT & MZC & DKT & MZC & DKT & MZC & DKT & MZC & DKT & \\
\hline 1 & 18.2 & 18.6 & 18.3 & 18.5 & 18.3 & 18.4 & 18.3 & 18.4 & 18.4 & 18.4 & 18.3 \\
\hline 2 & 72.1 & 77.8 & 72.9 & 75.5 & 73.1 & 74.7 & 73.3 & 74.2 & 73.4 & 74.1 & 73.5 \\
\hline 3 & 159.2 & 181.4 & 162.4 & 174.3 & 163.7 & 170.9 & 164.3 & 169.0 & 164.7 & 168.0 & 165.4 \\
\hline 4 & 276.6 & 327.5 & 285.1 & 315.4 & 288.7 & 308.4 & 290.6 & 304.2 & 291.7 & 301.6 & 294.1 \\
\hline 5 & 422.6 & 512.3 & 439.0 & 497.1 & 447 & 486.6 & 451.2 & 479.6 & 453.7 & 475.0 & 459.6 \\
\hline 6 & 773.3 & 800.7 & 773.1 & 981.2 & 776.9 & 926.2 & 777.9 & 880.6 & 597.1 & 640.9 & 661.8 \\
\hline
\end{tabular}

De las Tablas 2, 3 y 4 es posible apreciar que los valores de las frecuencias naturales de las placas calculadas con el elemento MZC y DKT son aproximaciones razonables del valor teórico otorgado por (30).

\section{Validación de la integración en el tiempo del registro de aceleraciones}

Se ha analizado la respuesta de un oscilador de 1 grado de libertad por medio de la solución exacta y posteriormente, mediante la implementación del método numérico de Newmark (1959). El oscilador que se ha considerado posee las propiedades y valores mostrados en la Tabla 1 .

La solución analítica de este oscilador está dada por la siguiente expresión:

$$
g(t)=\frac{\frac{F(t)}{K}}{\sqrt{\left(1-\left(\frac{\omega_{0}}{\omega}\right)^{2}\right)^{2}+\left(2 \xi \frac{\omega_{0}}{\omega}\right)^{2}}} \operatorname{sen}\left(\omega_{o}\right),
$$

donde $\omega_{0}$ es la frecuencia de la fuerza externa. Cabe destacar que esta expresión corresponde a la respuesta de un oscilador de 1 grado de libertad sometido a una carga sinusoidal monotónica, la cual no se puede extender 
a un registro aleatorio como lo es un registro sísmico. Finalmente, en la Figura 8 se muestra la comparación de ambas respuestas, en donde se aprecia que el resultado numérico es prácticamente igual a la solución exacta.

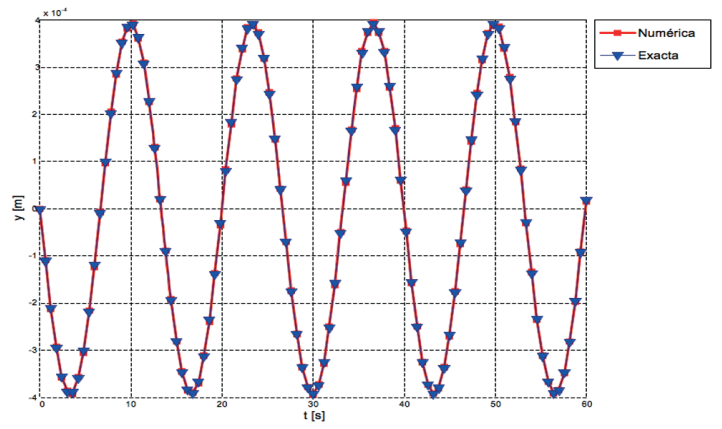

Figura 8: Respuesta otorgada por la solución exacta y numérica

\section{Análisis comparativo}

El análisis comparativo consiste en la obtención de las tensiones $\sigma_{x}, \sigma_{y}$ y $\tau_{x y}$, producto de la aplicación de fuerzas estáticas y sísmicas sobre 2 losas distintas. En el caso sísmico, la carga sobre la losa corresponde al registro de aceleraciones verticales del terremoto del 27/02/2010 (Colegio Concepción, San Pedro de la Paz) y para el caso estático es la carga que especifica la norma chilena NCh 1537Of.86, la cual en su tabla 3, especifica que la sobrecarga de uso uniformemente distribuida para pisos es de $2 \mathrm{kPa}$.

Para la comparación de ambos casos en el análisis sísmico de la losa, se ha considerado el punto 5.5.1 de la norma NCh 4330f96, la cual establece que para el cálculo de masas se deben considerar las cargas permanentes más un porcentaje de la sobrecarga de uso, que no podrá ser inferior a $25 \%$ en construcciones destinadas a la habitación o al uso público. La Tabla 6 resume las características del material y condiciones de apoyo de la losa. Los valores adoptados permanecen constantes durante el análisis. Cabe destacar que se consideró en el análisis el módulo de elasticidad estático del hormigón. Las características geométricas de las losas que se han analizado se muestran en las Figuras 9 y 10. La Tabla 7 entrega las cargas estáticas uniformemente repartida sobre la losa, sobrecarga, peso propio y carga total.

La carga sísmica sobre la losa corresponde al registro de aceleraciones verticales del terremoto del 27 de febrero del 2010 mostrado en la Figura 11.
Tabla 6: Valores de las propiedades de la losa

\begin{tabular}{|c|c|}
\hline Material & Hormigón armado (H-30) \\
\hline Condiciones de Borde & empotrada en todos sus bordes \\
\hline Espesor $[\mathrm{m}]$ & 0,14 \\
\hline$E\left[\frac{\mathrm{N}}{\mathrm{m}^{2}}\right]$ & $2,302581 \times 10^{10}$ \\
\hline$v$ & 0,2 \\
\hline$\rho$ Hormigón armado $\left[\frac{\mathrm{kg}}{\mathrm{m}^{3}}\right]$ & 2500 \\
\hline
\end{tabular}

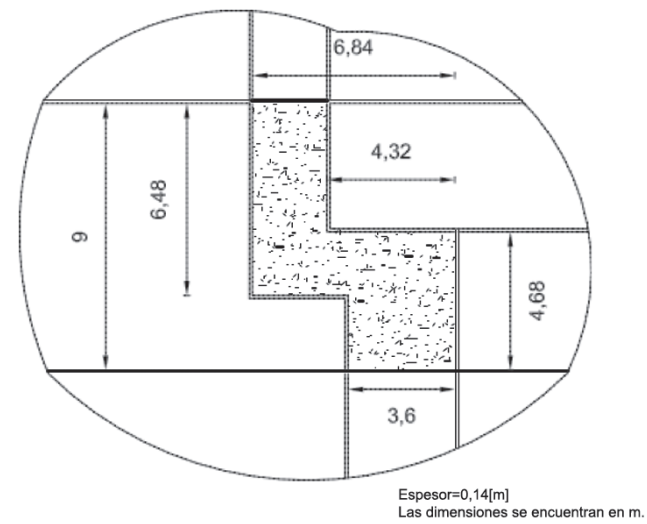

Figura 9: Vista en planta de la losa $\mathrm{N}^{\circ} 1$

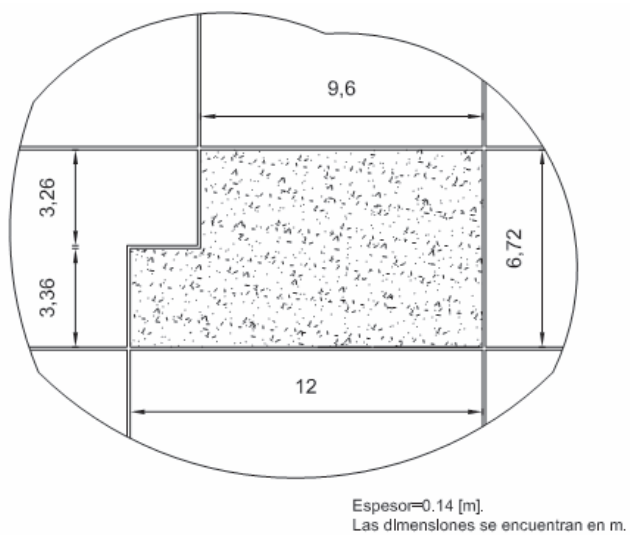

Figura 10: Vista en planta de la losa $\mathrm{N}^{\circ} 2$

Tabla 7: Cargas estáticas utilizadas en el análisis.

\begin{tabular}{|c|c|}
\hline Sobrecarga de uso & $2000\left[\frac{\mathrm{N}}{\mathrm{m}^{2}}\right]$ \\
\hline Peso propio losa & $3433.5\left[\frac{\mathrm{N}}{\mathrm{m}^{2}}\right]$ \\
\hline Carga Total sobre losa & $5433.5\left[\frac{\mathrm{N}}{\mathrm{m}^{2}}\right]$ \\
\hline
\end{tabular}

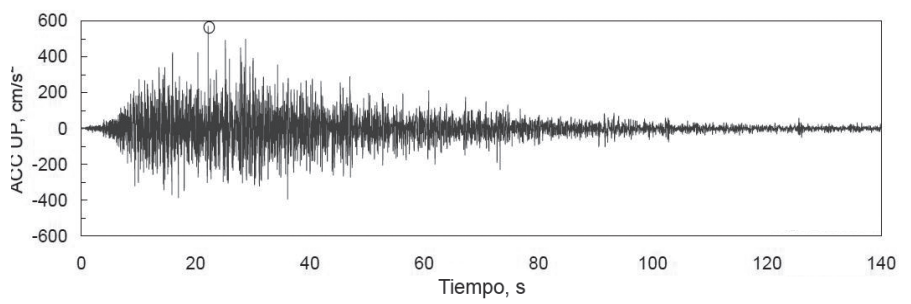

Figura 11: Registro de aceleraciones verticales del terremoto 27/02/2010 (Colegio Concepción, San Pedro de la Paz) 
Se debe tener en cuenta que en este trabajo, la masa de la losa corresponde a su masa como tal más un $100 \%$ de la sobrecarga de uso (según NCh433Of96), la cual es de 2 $\mathrm{kPa}$. En este caso se ha utilizado el $100 \%$ del valor de la sobrecarga debido a que es el caso más desfavorable.

Las Figuras 12, 13 y 14 muestran las distribuciones de tensiones a partir del elemento MZC y DKT para la losa 1 bajo carga estática.

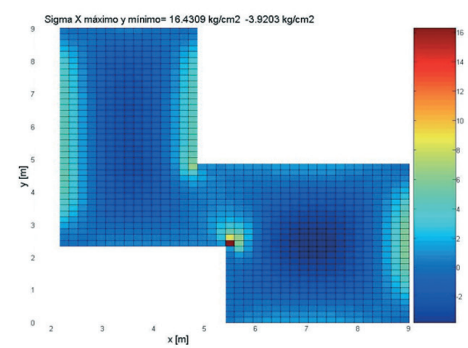

(a)

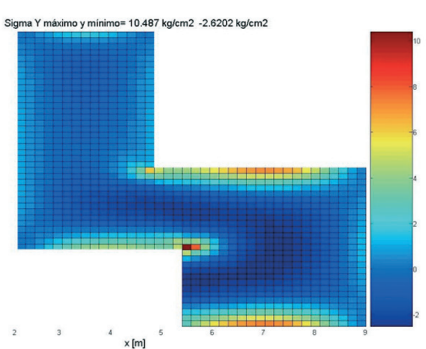

(b)
Figura 12: Distribuciones de tensiones en la losa 1 discretizada con elementos rectangulares, a) $\sigma_{x}$ y b) $\sigma_{y}$

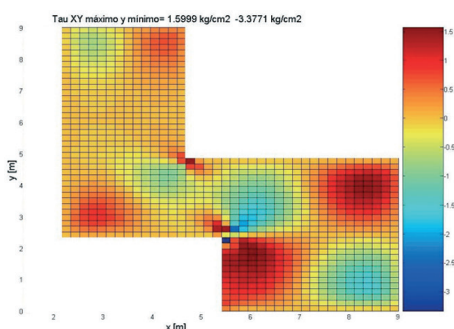

(a)

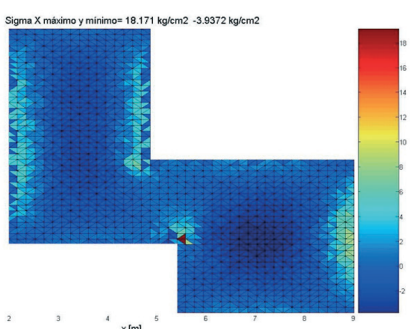

(b)
Figura 13: Distribuciones de tensiones, a) $\tau_{x y}$ en la losa 1 discretizada con elementos rectangulares y b) $\sigma_{x}$ en la losa 1 discretizada con elementos triangulares

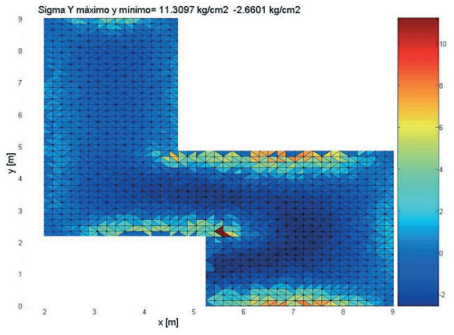

(a)

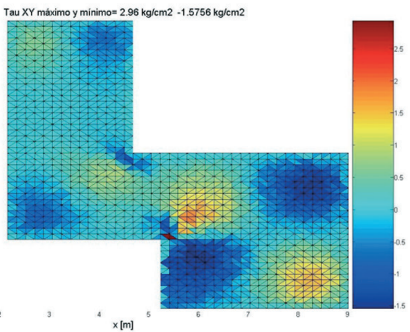

(b)
Figura 14: Distribuciones de tensiones en la losa 1 discretizada con elementos triangulares, a) $\sigma_{y}$ y b) $\tau_{x v}$

Las Figuras 15, 16 y 17 muestran las envolventes de tensiones a partir del elemento MZC y DKT para la losa 1 bajo carga sísmica. Notar que la escala en la dimensión horizontal $x$ ha sido reducida y que se han separado las tensiones positivas y negativas.

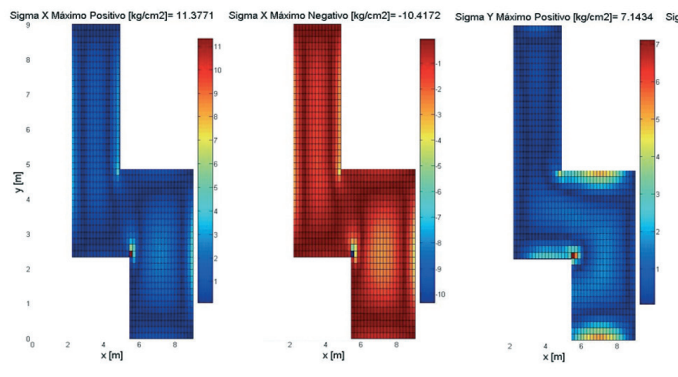

(a)

(b)

Figura 15: Envolventes de tensiones en la losa 1 discretizada con elementos rectangulares, a) $\sigma_{x}$ y b) $\sigma_{y}$

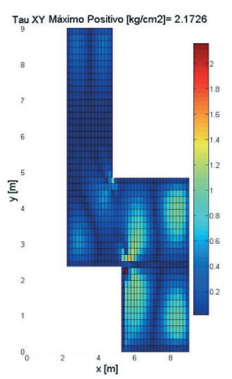

(a)

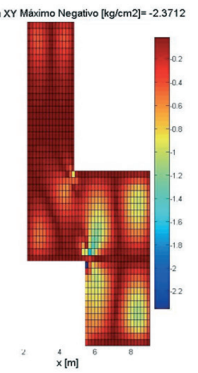

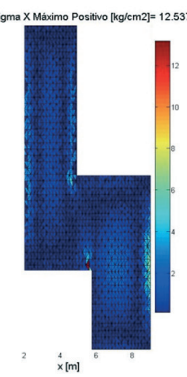

(b)
Figura 16: Envolventes de tensiones a) $\tau_{x y}$ en la losa 1 discretizada con elementos rectangulares y b) $\sigma_{x}$ en la losa 1 discretizada con elementos triangulares

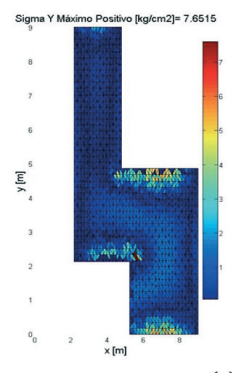

(a)

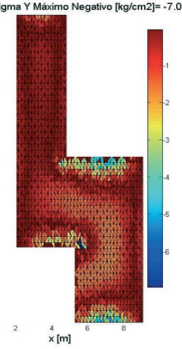

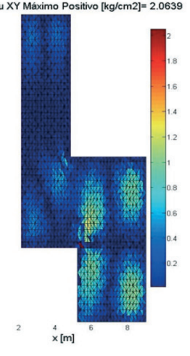

(b)

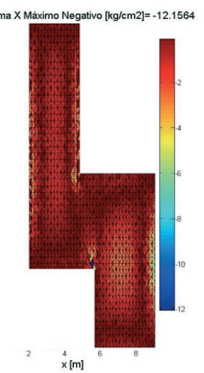

Figura 17: Envolventes de tensiones en la losa 1 discretizada con elementos triangulares, a) $\sigma_{y}$ y b) $\tau_{x y}$

Las tensiones máximas positivas sobre la losa 1 para el caso estático son mayores a las del caso sísmico. Sin embargo, se producen tensiones máximas negativas que son mayores a las del caso estático $\left(-\sigma_{\mathrm{X}},-\sigma_{\mathrm{y}},-\tau_{\mathrm{xy}}\right)$.

Las Figuras 18, 19 y 20 muestran las distribuciones de tensiones a partir del elemento MZC y DKT para la losa 2 bajo carga estática. 


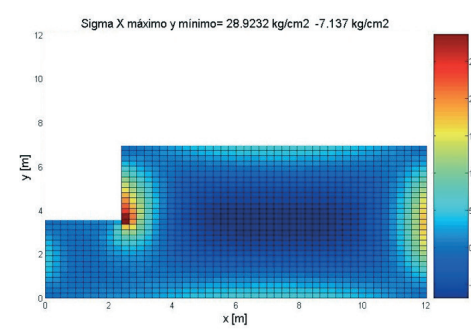

(a)

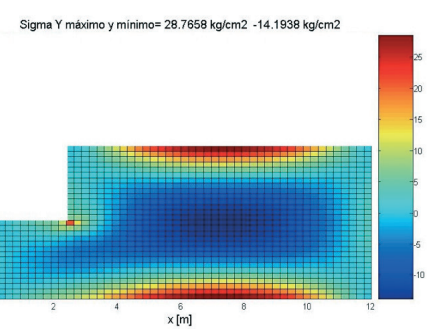

(b)
Figura 18: Distribuciones de tensiones en la losa 2 discretizada con elementos rectangulares, a) $\sigma_{x}$ y b) $\sigma_{y}$

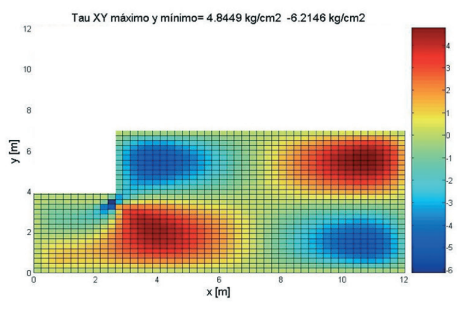

(a)

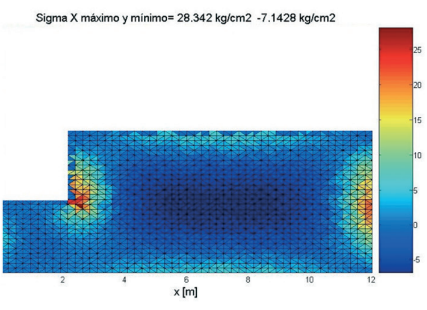

(b)
Figura 19: Distribuciones de tensiones, a) $\tau_{x y}$ en la losa 2 discretizada con elementos rectangulares y b) $\sigma_{x}$ en la losa 2 discretizada con elementos triangulares

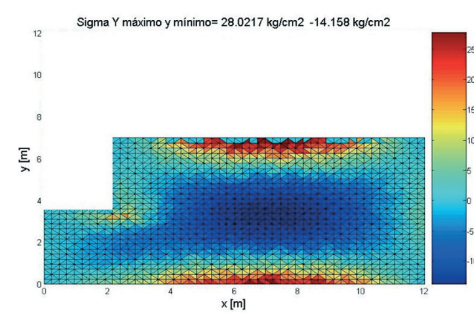

(a)

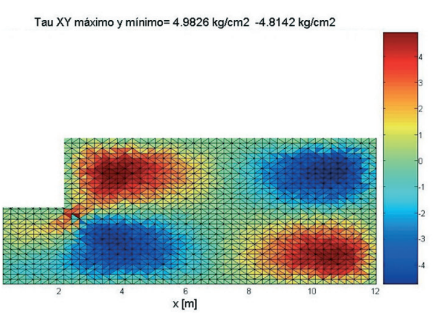

(b)
Figura 20: Distribuciones de tensiones en la losa 2 discretizada con elementos triangulares, a) $\sigma_{y}$ y b) $\tau_{x y}$

Las Figuras 21, 22 y 23 muestran las envolventes de tensiones a partir del elemento MZC y DKT para la losa 2 bajo carga sísmica. Notar que nuevamente se ha cambiado la escala y se han incluído las tensiones negativas.

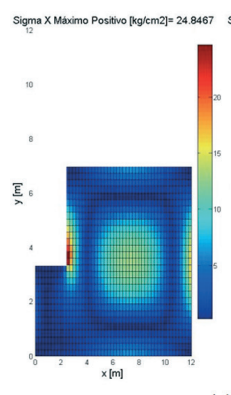

(a)

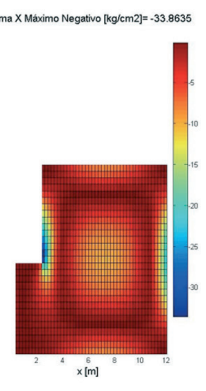

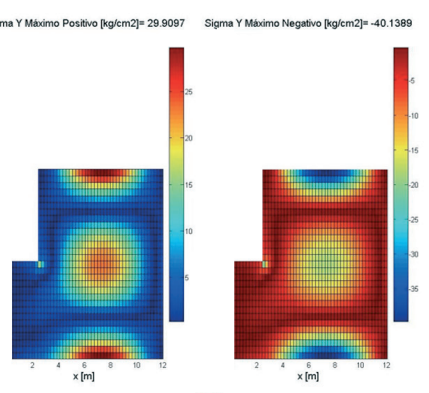

(b)
Figura 21: Envolventes de tensiones en la losa 2 discretizada con elementos rectangulares, a) $\sigma_{x} \mathrm{y}$ b) $\sigma_{y}$

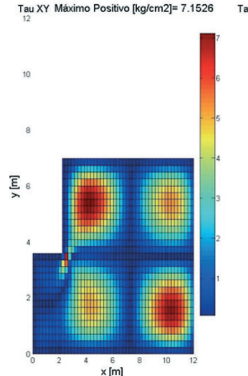

(a)

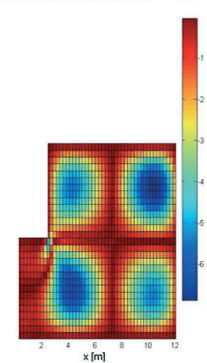

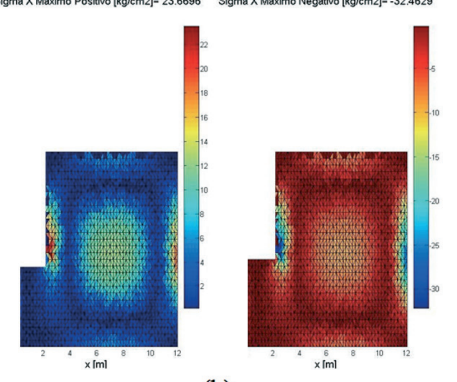

(b)
Figura 22: Envolventes de tensiones, a) $\tau_{x y}$ en la losa 2 discretizada con elementos rectangulares y b) $\sigma_{x}$ en la losa 2 discretizada con elementos triangulares

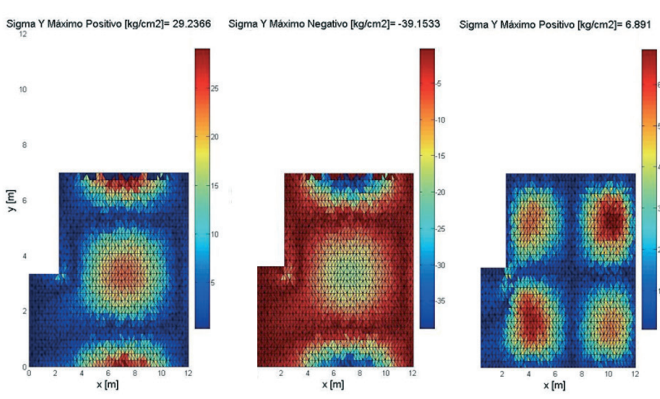

(a)

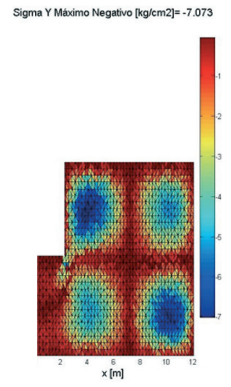

(b)

Figura 23: Envolventes de tensiones sobre la losa 2 discretizada con elementos triangulares, a) $\sigma_{y} y$ b) $\tau_{x y}$

En este caso tanto las tensiones máximas positivas como negativas que produce la aplicación del registro vertical del terremoto son siempre mayores a las que produce la aplicación de una carga estática vertical. Excepto en el caso de $\sigma_{x}$ positivo con elementos triangulares.

Los resultados con tensiones negativas de -4 MPa podrían explicar las grietas y desprendimientos de hormigón especialmente en las uniones de las losas con los muros o vigas tal como se mostró en la Figura 1.

Las periodos y frecuencias fundamentales calculados para la losa 1 y 2 son $0.031 \mathrm{~s}(198.9 \mathrm{rad} / \mathrm{s})$ y $0.104 \mathrm{~s}$ (60.4 rad/s) respectivamente. De esta manera el periodo fundamental de la losa 2 conlleva a aceleraciones espectrales mayores que la losa 1. Esto es porque la losa 2 es más flexible, ya que posee un periodo fundamental mayor. Por lo tanto, este aumento en la aceleración espectral se debe a que este terremoto en particular (en ese rango de periodos la aceleración espectral es ascendente), afecta mayormente a la losa 2 que a la losa 1. Esto explica que las tensiones provocadas por el registro vertical de aceleraciones 
aplicadas sobre la losa 2 superen a las que origina la carga estática aplicada sobre la misma losa, y para la losa 1 resulten menores.

\section{Conclusiones y recomendaciones}

Se han utilizado las teorías de placas basadas en la elasticidad lineal y la del movimiento de estructuras de 1 GDL para caracterizar el comportamiento sísmico de una losa de hormigón armado. Para ello se ha implementado un programa computacional en código MATLAB, el cual permite obtener desplazamientos verticales y tensiones $\pm \sigma_{\mathrm{X}}, \pm \sigma_{\mathrm{y}} \mathrm{y} \pm \tau_{\mathrm{X} y}$, producto de la aplicación de cargas estáticas y sísmicas. Se utilizaron los elementos finitos MZC y DKT, encontrándose no mayor diferencia en los resultados obtenidos al usar uno u otro elemento.

Las tensiones máximas en losas cuadradas y rectangulares empotradas surgen en los bordes de éstas, tanto para el caso estático como para el sísmico. La forma general de las distribuciones de tensiones en losas cuadradas y rectangulares son muy similares entre si, tanto para el caso estático como para el sísmico. Por otro lado, las fibras de una losa que habitualmente se encuentran en compresión (o tracción) bajo carga estática vertical, cambian temporalmente a tracción y compresión (compresión y tracción) durante la aplicación del registro de aceleraciones vertical del terremoto.

Los registros que posean una componente de aceleración vertical importante, como el del 27/02/2010 (San Pedro de la Paz), pueden provocar tensiones superiores a las que provocan cargas estáticas, como por ejemplo las cargas de diseño de la norma NCh 1537Of.86. Es por ello que se debería considerar la componente vertical en los análisis sísmicos, ya que sismos con aceleraciones verticales mayores a la del terremoto del 27/02/2010 podrían, eventualmente, provocar tensiones superiores a las que se consideran en los análisis para el diseño, tal como ocurre en las losas analizadas en este trabajo. Se recomienda en futuras investigaciones incluir a los muros en el análisis de aceleración vertical para así evaluar el efecto de interacción losa muro.

\section{Referencias}

Barrientos, S. (2010). Terremoto de Cauquenes 27 de Febrero del 2010. Informe técnico actualizado 27 de Mayo del 2010. Servicio Sismológico, Universidad de Chile.

Batoz, J.L., Bathe, K.J. and Ho, L. W. (1980). A study of threenode triangular plate bending elements. International Journal for Numerical Methods in Engineering 15, 1771-1812

Betanzo, R. (2010). Daños estructurales y lecciones del terremoto del 27/f en el Gran Concepción. Obras y Proyectos 8, 59-75

Boroschek, R., Soto, P. and León, R. (2010). Maule Region Earthquake. February 27, 2010, $\mathrm{M}_{\mathrm{w}}=8.8$. Renadic report 10/08 Rev. 2. Universidad de Chile

Chakraverty, S. (2009). Vibration of plates. Crc Press Taylor \& Francis Group. USA

IDIEM (2010). Informe Inspección Post Sismo del 27 de Febrero del 2010, Edificio Don Manuel. Informe ejecutivo $\mathrm{N}^{\circ}$ 595408

Luo, Z. and Hutton, S.G. (2002). Formulation of a three-node traveling triangular plate element subjected to gyroscopy and inplane forces. Computers \& Structures 80, 1935-1944.

Melosh, R.J. (1963). Basis for derivation of matrices for the direct stiffness method. Journal of the American Institute of Aeronautics and Astronautic AIAA 1, no. 7, 1631 - 1637

NCh 4330f.96. Diseño sísmico de edificios. Instituto Nacional de Normalización, Chile

NCh 1537Of.86. Diseño estructural de edificios, cargas permanentes y sobrecargas de uso. Instituto Nacional de Normalización, Chile

Newmark, N.M. (1959). A method of computation of structural dynamics. Proceedings American Society of Civil Engineers 85, EM3, 67-94

Novoa, N. (2001). Análisis de cargas críticas en estructuras de cáscara delgada. Memoria para optar al título de Ingeniero Civil. Universidad de Concepción

Oñate, E. (1992). Cálculo de Estructuras por el Método de Elementos Finitos. Centro Internacional de Métodos Numéricos en Ingeniería, Primera Edición. España

Paz, M. (1992). Dinámica estructural, teoría y cálculo. Editorial Reverté. Tercera edición, España

Timoshenko, S. (1937). Vibration problems in engineering. Van Nostrand Company, Inc., USA

Zienkiewicz, 0. C. and Cheung, Y. K. (1967). The finite element method in structural and continuum mechanics. McGraw-Hill 